\title{
Calculation of Helium nuclei in quenched lattice QCD
}

\section{Takeshi Yamazaki* for PACS-CS Collaboration}

Center for Computational Physics, University of Tsukuba, Tsukuba, Ibaraki 305-8577, Japan yamazaki@kmi.nagoya-u.ac.jp

We present results for the binding energies for ${ }^{4} \mathrm{He}$ and ${ }^{3} \mathrm{He}$ nuclei calculated in quenched lattice QCD at the lattice spacing of $a=0.128 \mathrm{fm}$ with a heavy quark mass corresponding to $m_{\pi}=0.8$ $\mathrm{GeV}$. Enormous computational cost for the nucleus correlation functions is reduced by avoiding redundancy of equivalent contractions stemming from permutation symmetry of protons or neutrons in the nucleus and various other symmetries. To distinguish a bound state from an attractive scattering state, we investigate the volume dependence of the energy difference between the ground state energy of the nucleus channel and the free multi-nucleon states by changing the spatial extent of the lattice from $3.1 \mathrm{fm}$ to $12.3 \mathrm{fm}$. A finite energy difference left in the infinite spatial volume limit leads to the conclusion that the measured ground states are bounded. It is also encouraging that the measured binding energies and the experimental ones show the same order of magnitude.

The XXVIII International Symposium on Lattice Field Theory, Lattice2010

June 14-19, 2010

Villasimius, Italy

${ }^{*}$ Present address: Kobayashi-Maskawa Institute for the Origin of Particles and the Universe, Nagoya University, Naogya, Aichi 464-8602, Japan 


\section{Introduction}

The atomic nuclei have been historically treated as collections of protons and neutrons. The great success of the nuclear shell model since 1949 [1], 2], explaining the nuclear magic numbers and detailed spectroscopy, has established that protons and neutrons are very good effective degrees of freedom at the nuclear energy scale of a few MeV. Nonetheless, 60 years later, we know for certain that protons and neutrons are made of quarks and gluons whose laws are governed by QCD. It is a great challenge to quantitatively understand the structure and property of known nuclei based on the first principle of QCD. This direct approach will be more important and indispensable if we are to extract reliable predictions for experimentally unknown nuclei in the neutron rich regions of the nuclear chart. In this article we address the fundamental question in the research in this direction, namely the binding energies of nuclei.

Lattice QCD study of multi-baryon states goes back a long time, starting with $\mathrm{H}$ dibaryon [3] in the 80's [4] and deuteron in the early 90's [5]. More recently, exploration of three baryon states began [6]. So far, however, there is no established evidence supporting bound state formation in these channels. An exception is a model study in the strong coupling limit of lattice QCD [ד].

We attempt to go a step further in mass number and examine the helium nuclei, especially ${ }^{4} \mathrm{He}$ with the mass number $A=4$. Besides the obvious physical interest as the first natural element beyond hydrogen, it is also the system where technical difficulties of fermion contractions specific to nuclei with a large mass number appear in a non-trivial way. On the other hand, the binding energy drops down to a large value of $\Delta E=28.3 \mathrm{MeV}$ for the ${ }^{4} \mathrm{He}$ nucleus, making us hopeful that observing the bound state nature might be easier than the lighter nuclei.

The organization of this article is as follows. In Sec. 2 we review previous studies for bound states in multi-baryon systems from lattice QCD. The computational issues with studies of multibaryon states and their solutions employed in this work are briefly explained in Sec. 3 . The simulation details and the results for the ${ }^{4} \mathrm{He}$ and ${ }^{3} \mathrm{He}$ channels are presented in Sec. 4 . A brief summary and a look toward future are given in Sec. 5. The results in this article have been reported in Ref. [8].

\section{Historical perspective}

Bound states in multi-baryon systems have been investigated by several studies in lattice QCD. For systems with two baryons, the first study was the search for the $\mathrm{H}$ dibaryon. According to Jaffe [3] the $\mathrm{H}$ dibaryon having strangeness $S=-2$ and isospin $I=0$ channel was expected to have a large binding energy of $O(100) \mathrm{MeV}$. Most of the quenched lattice QCD studies [ [ , 9, 10, 11, 12] concluded that the $\mathrm{H}$ dibaryon bound state does not exist. Recently NPLQCD Collaboration observed a small, negative energy shift, $E_{\Lambda \Lambda}-2 m_{\Lambda}=-4.1(1.2)(1.4) \mathrm{MeV}$ [13], in this channel. They concluded, however, that the evidence is not strong enough to establish the existence of the $\mathrm{H}$ dibaryon, and that further study is necessary with different volumes. The latter point is related to the computational problem of the nucleus calculation, which we will discuss in the next section.

Deuteron is a bound state of two nucleons in the ${ }^{3} S_{1}$ and $I=0$ channel. Nucleon-nucleon scattering in this channel and also in the ${ }^{1} S_{0}$ channel was first studied in quenched QCD [5, 14]. This work was followed by a partially-quenched mixed action [15] and $N_{f}=2+1$ anisotropic Wilson 

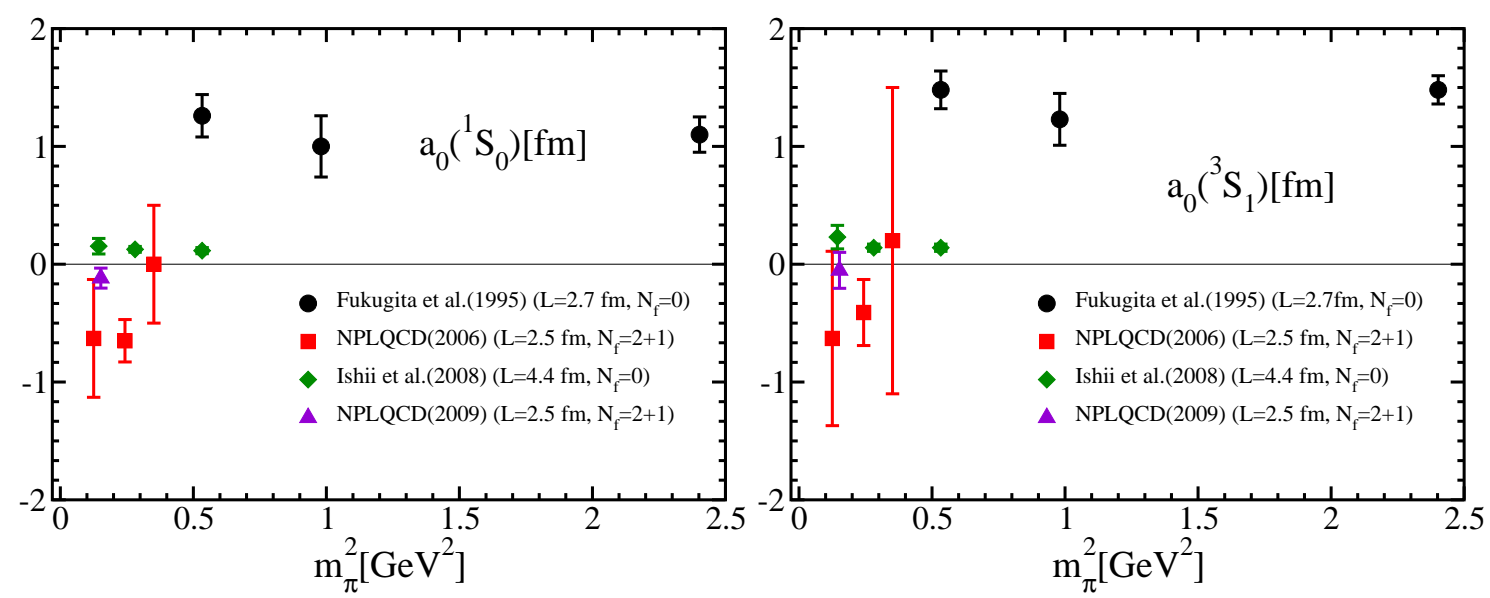

Figure 1: Scattering length for ${ }^{1} S_{0}$ (left) and ${ }^{3} S_{1}$ (right) channels. Circle, square, diamond, and triangle denote results for quenched [5, 14], mixed action [15], two-nucleon wave function [16], and anisotropic Wilson [13] calculations, respectively.

action [13] simulations. Extraction of nuclear force between two nucleons has been investigated in quenched and 2+1 flavor QCD [16, 17, 18]. Results for the scattering lengths $a_{0}$ from these studies are summarized in Fig. 1. The scattering lengths in the two channels are almost identical in each group. The results, however, have large discrepancies between the groups. An even more problematic issue is that the absolute value of the lattice results is much smaller than the experimental values, $a_{0}=23.7 \mathrm{fm}$ and $a_{0}=-5.47 \mathrm{fm}$ for the ${ }^{1} S_{0}$ and ${ }^{3} S_{1}$ channels, respectively. The lattice results do not show strong dependence on the pion mass at the region where the calculations were carried out, $m_{\pi} \gtrsim 0.3 \mathrm{GeV}$. In order to explain the experimental values, the scattering lengths have to vary significantly when calculations near the physical quark mass are carried out in future. We should also note that all these studies assumed that the deuteron state is not bound for the heavy pion mass employed in the calculations.

Recently not only two-baryon systems but also three-baryon systems have been investigated using lattice QCD. NPLQCD Collaboration has tried a feasibility study of three-baryon systems focusing on the $\Xi^{0} \Xi^{0} n$ and the $n n p$ (triton) channels. They found both interactions to be repulsive [6, 19], which indicates that the triton is not bound for the parameters taken for the calculation.

In this conference several studies of two- and three-baryon systems were reported. HALQCD Collaboration studied (i) the energy dependence of the nuclear force [20], (ii) the nuclear force in the flavor $\mathrm{SU}(3)$ limit [21], (iii) extraction of the two-baryon forces in a coupled channel with the variational method [22, 23], and (iv) an exploratory study of extraction of the three-nucleon force [24]. In multi-meson systems, NPLQCD Collaboration proposed a recursion relation approach for multi-meson correlation functions [25, 26] to largely reduce the computational cost of the correlation functions.

\section{Computational issues with nuclei}

There are several computational difficulties in the calculation of the multi-baryon bound state in lattice QCD. They are : 1) exponential increase of statistical error, 2) factorial growth of fermion 
Wick contractions, and 3) identification of bound state. While we avoid the first one by an unphysical heavy quark mass, we propose solutions for the second and third problems. Let us discuss each in turn.

\subsection{Exponential increase of statistical error}

An estimate of the statistical noise to signal ratio for the correlation function of the nucleus consisting of $N_{N}$ nucleons is known [27] to be proportional to

$$
\frac{1}{\sqrt{N_{\text {meas }}}} \exp \left(N_{N}\left[m_{N}-\frac{3}{2} m_{\pi}\right] t\right)
$$

where $m_{\pi}$ and $m_{N}$ are the masses of the pion and nucleon, respectively, $N_{\text {meas }}$ is the number of measurement, and $t$ is the separation between the source and sink time slices. The statistical error exponentially increases as the number of nucleon increases as well as when the quark mass decreases. We aim to treat helium nuclei in this work, so that $N_{N}$ is fixed to four and three for ${ }^{4} \mathrm{He}$ and ${ }^{3} \mathrm{He}$ channels, respectively. Since our main aim is to explore nucleus calculations, and since the difficulty of controlling statistical fluctuations toward the region of lighter pion mass is well known, we use the heavy quark mass corresponding to $m_{\pi}=0.8 \mathrm{GeV}$. Even then we had to carry out $O\left(10^{3}\right)$ measurements.

While this strategy would be acceptable for a feasibility test of calculation of nucleus, we need novel methods to solve this problem for a more realistic calculation near the physical quark mass. We leave this task in future.

\subsection{Factorial growth of Wick contractions}

Another computational problem for multi-nucleon systems is a factorially large number of Wick contractions of quark-antiquark fields required for evaluations of the nucleus correlation functions. A naive counting would give $\left(2 N_{p}+N_{n}\right) !\left(2 N_{n}+N_{p}\right)$ ! for a nucleus composed of $N_{p}$ protons and $N_{n}$ neutrons, which quickly becomes prohibitively large beyond three-nucleon systems, e.g., 2880 for ${ }^{3} \mathrm{He}$ and 518400 for ${ }^{4} \mathrm{He}$.

This number, however, contains equivalent contractions under the permutation symmetry in terms of the protons or the neutrons in the interpolating operator. We can reduce the computational cost by avoiding the redundancy. In the case of the ${ }^{4} \mathrm{He}$ nucleus which consists of the same number of protons and neutrons, the isospin symmetry also helps us reduce the necessary contractions. After a scrutiny of the remaining equivalent contractions by a computer we find that only 1107 (93) contractions are required for the ${ }^{4} \mathrm{He}\left({ }^{3} \mathrm{He}\right)$ nucleus correlation function. We have made a numerical test that the result with the reduced contractions reproduces the one with the full contractions on a configuration.

For an additional technique to save the computational cost of the nucleus correlation functions, we make a block of three quark propagators where a nucleon operator with zero spatial momentum is constructed in the sink time slice. In this procedure we can incorporate the permutation symmetry of two up (down) quarks in a proton (neutron) sink operator. This is a simple trick to calculate $2^{N_{N}}$ contractions simultaneously. We also prepare several combinations of the two blocks which are useful for the construction of the nucleus correlators. 


\begin{tabular}{cccccc}
\hline \hline$L$ & $N_{\text {conf }}$ & $N_{\text {meas }}$ & accept.(\%) & $m_{\pi}[\mathrm{GeV}]$ & $m_{N}[\mathrm{GeV}]$ \\
\hline 24 & 2500 & 2 & 93 & $0.8000(3)$ & $1.619(2)$ \\
48 & 400 & 12 & 93 & $0.7999(4)$ & $1.617(2)$ \\
96 & 200 & 12 & 68 & $0.8002(3)$ & $1.617(2)$ \\
\hline \hline
\end{tabular}

Table 1: Number of configurations ( $\left.N_{\text {conf }}\right)$, number of measurements on each configuration $\left(N_{\text {meas }}\right)$, acceptance rate in the HMC algorithm, pion mass $\left(m_{\pi}\right)$ and nucleon mass $\left(m_{N}\right)$.

\subsection{Identification of bound state}

A general issue with numerical calculations for exploring bound state formation is to distinguish the physical binding energy from the energy shift due to the finite volume effect in the attractive scattering system [28, 29, 30]. The problem is made more difficult for nuclei because the binding energy $\Delta E$ of the nucleus consisting of $N_{N}$ nucleons with the mass $m_{N}$ is very tiny compared with the mass $M$ of the nucleus: $\Delta E / M \sim O\left(10^{-3}\right)$ with $\Delta E=N_{N} m_{N}-M$.

One way to solve the problem is to investigate the volume dependence of the measured energy shift: In the attractive scattering system the energy shift is proportional to $1 / L^{3}$ at the leading order in the $1 / L$ expansion [28, 31], while the physical binding energy remains at a finite value at the infinite spatial volume limit.

If the volume is not large enough, it is difficult to distinguish a constant from a $1 / L^{3}$ behavior in the energy shift. Thus, in our simulation we employ large volumes as much as possible, and choose three spatial extents corresponding to 3.1, 6.1 and $12.3 \mathrm{fm}$. The largest two volumes are much larger than those employed in current numerical simulations. They should provide sufficient room for the helium nuclei.

\section{A quenched calculation of Helium nuclei}

\subsection{Simulation details}

We carry out calculations on quenched configurations generated with the Iwasaki gauge action [32] at $\beta=2.416$ whose lattice spacing is $a=0.128 \mathrm{fm}$ determined with $r_{0}=0.49 \mathrm{fm}$ as an input [33]. We employ the HMC algorithm with the Omelyan-Mryglod-Folk integrator [34, 35]. The step size is chosen to yield reasonable acceptance rate presented in Table 1. We take three lattice sizes, $L^{3} \times T=24^{3} \times 64,48^{3} \times 48$ and $96^{3} \times 48$, to investigate the spatial volume dependence of the energy difference between the ground state of the nucleus channel and the free multi-nucleon states. The physical spatial extents are 3.1, 6.1 and $12.3 \mathrm{fm}$, respectively.

We use the tadpole improved Wilson action with $c_{\mathrm{SW}}=1.378$ [33]. As discussed in the previous section, since it becomes harder to obtain a reasonable signal-to-noise ratio at lighter quark masses for the multi-nucleon system, we employ a heavy quark mass at $\kappa=0.13482$ which gives $m_{\pi}=0.8 \mathrm{GeV}$ for the pion mass and $m_{N}=1.6 \mathrm{GeV}$ for the nucleon mass. Statistics are increased by repeating the measurement of the nucleus correlation functions with the source points in different time slices on each configuration. The numbers for the configurations and the measurements on each configuration are summarized in Table 1. We separate 100 trajectories between each measurement with $\tau=1$ for the trajectory length. The errors are estimated by the jackknife analysis choosing 200 trajectories for the bin size. 
The quark propagators are solved with the periodic boundary condition in all the spatial and temporal directions, and using the exponentially smeared source

$$
q^{\prime}(\vec{x}, t)=\sum_{\vec{y}} A e^{-B|\vec{x}-\vec{y}|} q(\vec{y}, t)
$$

after the Coulomb gauge fixing. $q$ is the quark field at the source time slice and $A, B$ are the smearing parameters. On each volume we employ two sets of the smearing parameters: $(A, B)=(0.5,0.5)$ and $(0.5,0.1)$ for $L=24$ and $(0.5,0.5)$ and $(1.0,0.4)$ for $L=48$ and 96. Effective mass plots with different sources, which are shown later, help us confirm the ground state in the nucleus channel. Hereafter the first and the second smearing parameter sets are referred to as " $S_{1,2}$ ", respectively.

The interpolating operator for the proton is defined as $p_{\alpha}=\varepsilon_{a b c}\left(\left[u_{a}\right]^{t} C \gamma_{5} d_{b}\right) u_{c}^{\alpha}$ where $C=\gamma_{4} \gamma_{2}$ and $\alpha$ and $a, b, c$ are the Dirac index and the color indices, respectively. The neutron operator $n_{\alpha}$ is obtained by replacing $u_{c}^{\alpha}$ by $d_{c}^{\alpha}$ in the proton operator. To save the computational cost we use the nonrelativistic quark operator, in which the Dirac index is restricted to upper two components.

The ${ }^{4} \mathrm{He}$ nucleus has zero total angular momentum and positive parity $\mathrm{J}^{P}=0^{+}$with the isospin singlet $I=0$. We employ the simplest ${ }^{4} \mathrm{He}$ interpolating operator with the zero orbital angular momentum $L=0$, and hence $J=S$ with $S$ being the total spin. Such an operator was already given long time ago in Ref. [36],

$$
{ }^{4} \mathrm{He}=(\bar{\chi} \eta-\chi \bar{\eta}) / \sqrt{2},
$$

where

$$
\begin{aligned}
& \chi=([+-+-]+[-+-+]-[+--+]-[-++-]) / 2 \\
& \bar{\chi}=([+-+-]+[-+-+]+[+--+]+[-++-]-2[++--]-2[--++]) / \sqrt{12}(
\end{aligned}
$$

with $+/-$ being up/down spin of each nucleon. $\eta, \bar{\eta}$ are obtained by replacing $+/-$ in $\chi, \bar{\chi}$ by $p / n$ for the isospin. Each nucleon in the sink operator is projected to have zero spatial momentum.

We also calculate the correlation function of the ${ }^{3} \mathrm{He}$ nucleus whose quantum numbers are $J^{P}=\frac{1}{2}^{+}, I=\frac{1}{2}$ and $I_{z}=\frac{1}{2}$. We employ the interpolating operator in Ref. [37],

$$
{ }^{3} \mathrm{He}=\left(\left|p_{-} n_{+} p_{+}\right\rangle-\left|p_{+} n_{+} p_{-}\right\rangle+\left|n_{+} p_{+} p_{-}\right\rangle-\left|n_{+} p_{-} p_{+}\right\rangle+\left|p_{+} p_{-} n_{+}\right\rangle-\left|p_{-} p_{+} n_{+}\right\rangle\right) / \sqrt{6},
$$

with the zero momentum projection on each nucleon in the sink operator.

\section{2 ${ }^{4}$ He channel}

Let us first present the results for the ${ }^{4} \mathrm{He}$ channel. The left panel of figure 2 shows the effective mass plots of the ${ }^{4} \mathrm{He}$ nucleus correlators with the $S_{1,2}$ sources on the $(6.1 \mathrm{fm})^{3}$ spatial volume. We find clear signals up to $t \approx 12$, beyond which statistical fluctuation dominates. The effective masses with the different sources show a reasonable agreement in the plateau region. The consistency is also shown in the exponential fit results in the plateau region as presented by the solid lines in the figure.

In order to determine the energy shift $\Delta E_{L}$ precisely, we define the ratio of the ${ }^{4} \mathrm{He}$ nucleus correlation function divided by the fourth power of the nucleon correlation function,

$$
R_{4} \mathrm{He}(t)=\frac{G_{4} \mathrm{He}_{\mathrm{e}}(t)}{\left(G_{N}(t)\right)^{4}},
$$



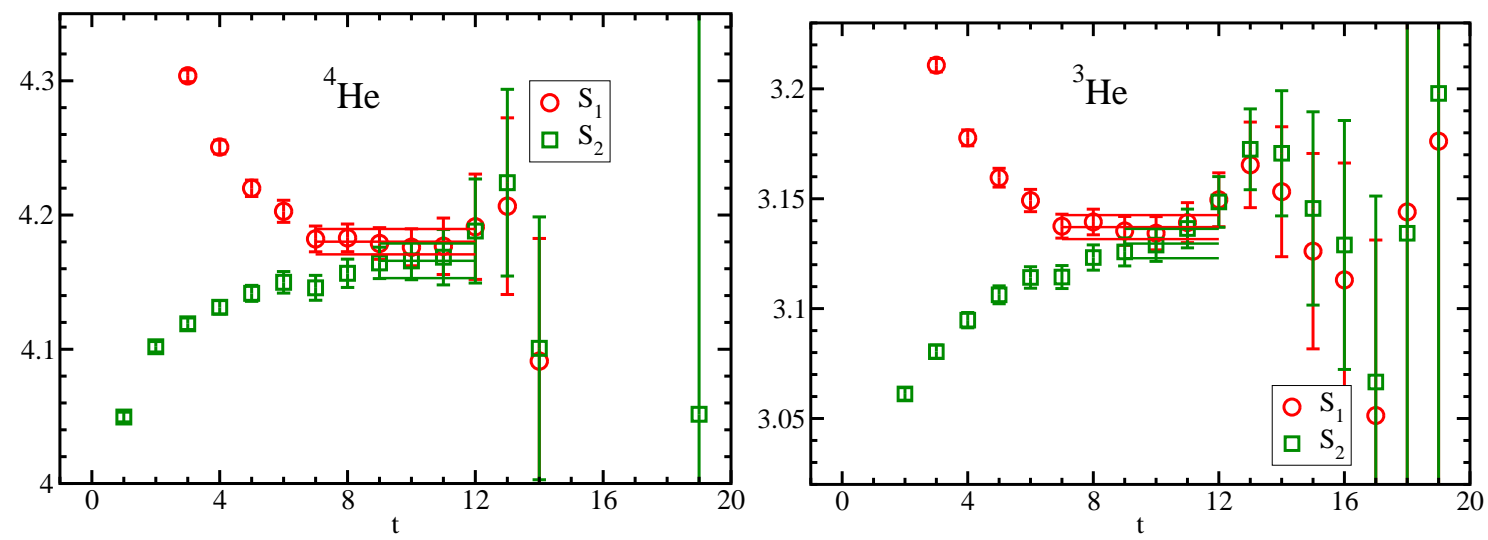

Figure 2: Effective masses for ${ }^{4} \mathrm{He}$ (left) and ${ }^{3} \mathrm{He}$ (right) correlation functions with $S_{1}$ (circle) and $S_{2}$ (square) sources at spatial extent of $6.1 \mathrm{fm}$. Fit results with one standard deviation error band are expressed by solid lines.
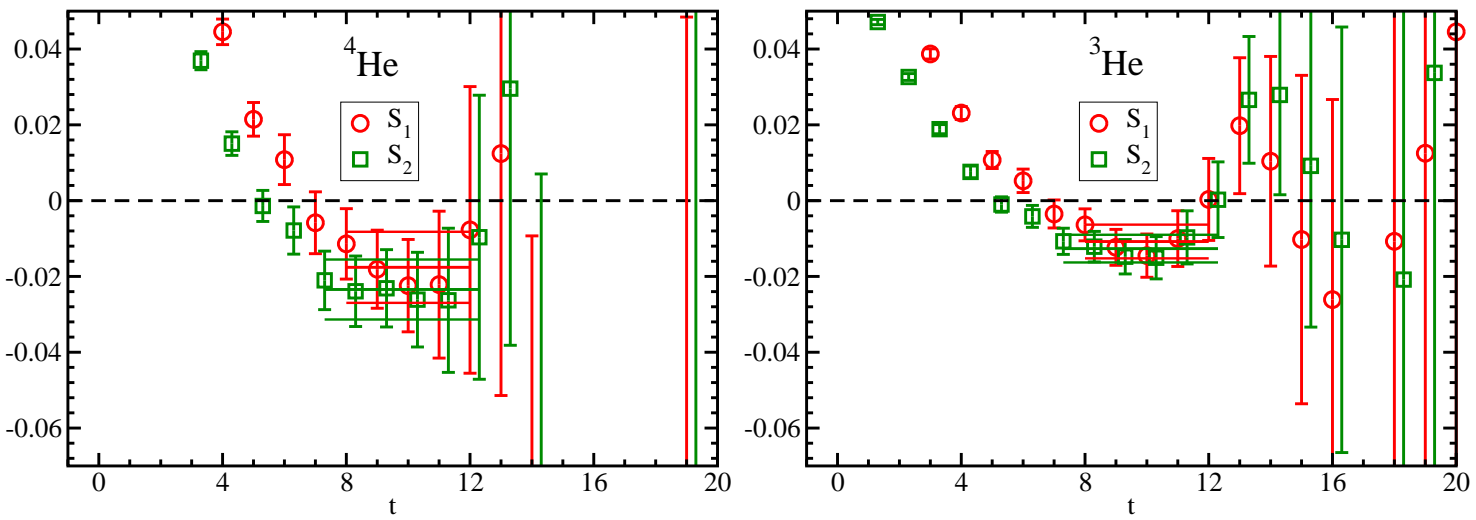

Figure 3: Effective energy shifts for ${ }^{4} \mathrm{He}$ (left) and ${ }^{3} \mathrm{He}$ (right) channels in a convention of $-\Delta E_{L}^{\text {eff }}$ with $S_{1}$ (circle) and $S_{2}$ (square) sources at spatial extent of $6.1 \mathrm{fm}$. Square symbols are slightly shifted to positive direction in horizontal axis for clarity. Fit results with one standard deviation error band are expressed by solid lines.

where $G_{4} \mathrm{He}(t)$ and $G_{N}(t)$ are obtained with the same source. The effective energy shift is extracted as

$$
-\Delta E_{L}^{\mathrm{eff}}=\ln \left(\frac{R(t)}{R(t+1)}\right),
$$

once the ground states dominate in both of the correlators. In the left panel of Fig. 3 we present time dependence of $-\Delta E_{L}^{\mathrm{eff}}$ for the $S_{1,2}$ sources, both of which show negative values beyond the error bars in the plateau region of $8 \leq t \leq 11$. Note that this plateau region is reasonably consistent with that for the effective mass of the ${ }^{4} \mathrm{He}$ nucleus correlators in the left panel of Fig. 2. The signals of $-\Delta E_{L}^{\text {eff }}$ are lost beyond $t \approx 12$ because of the large fluctuations in the ${ }^{4} \mathrm{He}$ nucleus correlator. We determine $\Delta E_{L}$ by exponential fits of the ratios in the plateau region, $t=8-12$ for $S_{1}$ and $t=7-12$ for $S_{2}$, respectively. We estimate a systematic error of $\Delta E_{L}$ from the difference of the central values of the fit results with the minimum or maximum time slice changed by \pm 1 .

Table 2 summarizes the numerical values of the energy shift $\Delta E_{L}$ at three spatial volumes, where the statistical and systematic errors are presented in the first and second parentheses, respec- 

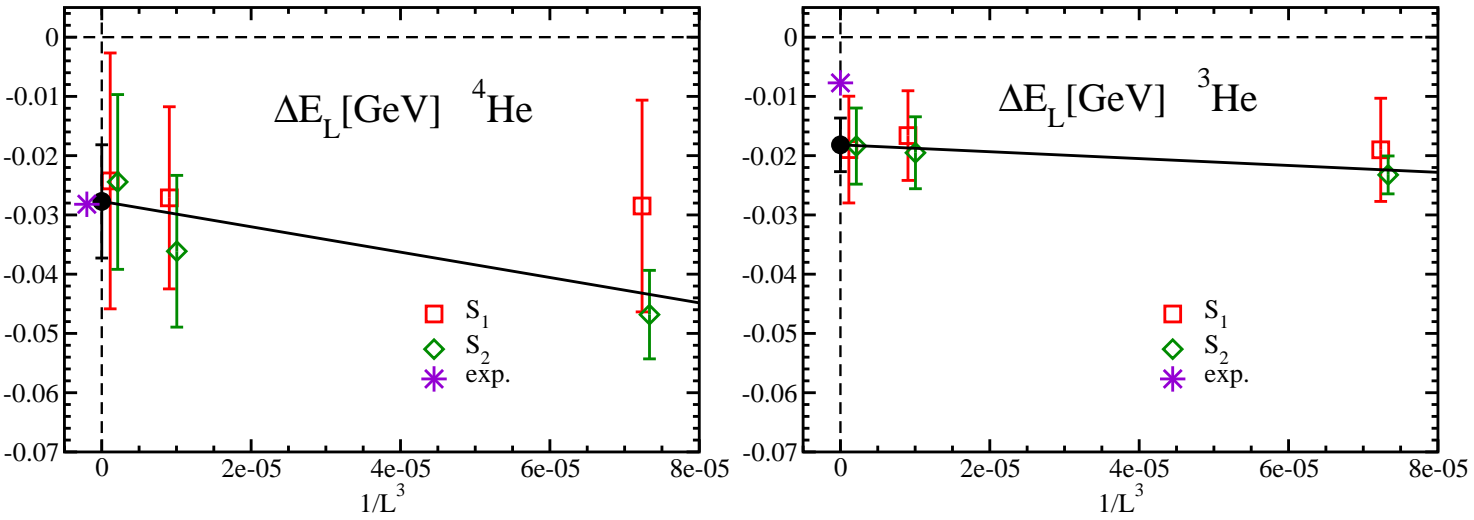

Figure 4: Spatial volume dependences for $-\Delta E_{L}=M-N_{N} m_{N}$ in $\mathrm{GeV}$ units for ${ }^{4} \mathrm{He}$ (left) and ${ }^{3} \mathrm{He}$ (right) nuclei with $S_{1}$ (open square) and $S_{2}$ (open diamond) sources. Statistical and systematic errors are added in quadrature. Diamond symbols are slightly shifted to positive direction in horizontal axis for clarity. Extrapolated results to the infinite spatial volume limit (filled circle) and experimental values (star) are also presented.

tively. The volume dependence of $\Delta E_{L}$ is plotted as a function of $1 / L^{3}$ in the left panel of Fig. 4 . The errors in the figure are evaluated from the statistical and systematic errors added in quadrature. In the following discussions in this subsection we use the combined error. The results for the $S_{1,2}$ sources are consistent within the error bars. We observe little volume dependence for $\Delta E_{L}$ indicating a bound state, rather than the $1 / L^{3}$ dependence expected for a scattering state, for the ground state in the ${ }^{4} \mathrm{He}$ channel.

The physical binding energy $\Delta E$ defined in the infinite spatial volume limit is extracted by a simultaneous fit of the data for the $S_{1,2}$ sources employing a fit function of $\Delta E+C / L^{3}$ with $\Delta E$ and $C$ free parameters. The $1 / L^{3}$ term is added to allow for contamination of scattering states. A systematic error is estimated from the difference of the central values of the fit results using the data with the different fit ranges in the determination of $\Delta E_{L}$. The result for $\Delta E$ is $0.0180(62)$ in lattice units, which is $2.9 \sigma$ away from zero as shown in the left panel of Fig. 7 . We also try a pure bound state fit allowing for an exponentially small finite size correction: $\Delta E$ and $\Delta E+C_{1} e^{-C_{2} L}$ with $\Delta E$ and $C_{1,2}$ free parameters. We find all the results are in agreement with reasonable values of $\chi^{2}$.

Based on these analyses we conclude that the ground state of the measured four-nucleon system is bounded. An encouraging finding is that $\Delta E=27.7(9.6) \mathrm{MeV}$ with $a^{-1}=1.54 \mathrm{GeV}$ agrees with the experimental value of $28.3 \mathrm{MeV}$. However, we do not intend to stress the consistency because our calculation is performed at the unphysically heavy pion mass, $m_{\pi}=0.8 \mathrm{GeV}$, and the electromagnetic interactions and the isospin symmetry breaking effects are neglected.

\section{$4.3{ }^{3} \mathrm{He}$ channel}

The results of effective mass and the effective energy shift for the ${ }^{3} \mathrm{He}$ channel with the $S_{1,2}$ sources are shown in the right panel of Figs. 目 and B, respectively. The statistical error is slightly smaller than those for the ${ }^{4} \mathrm{He}$ channel. We determine $\Delta E_{L}$ for the ${ }^{3} \mathrm{He}$ channel as in the ${ }^{4} \mathrm{He}$ channel, whose results are presented in the right panel of Fig. 7 and Table 2 . The trend of the volume dependence is similar to the ${ }^{4} \mathrm{He}$ channel case. A simultaneous fit of the data for the $S_{1,2}$ 


\begin{tabular}{ccccc}
\hline \hline$L$ & \multicolumn{4}{c}{$\Delta E_{L}[\mathrm{MeV}]$} \\
& ${ }^{4} \mathrm{He}\left(S_{1}\right)$ & ${ }^{4} \mathrm{He}\left(S_{2}\right)$ & ${ }^{3} \mathrm{He}\left(S_{1}\right)$ & ${ }^{3} \mathrm{He}\left(S_{2}\right)$ \\
\hline 24 & $28(14)(11)$ & $46.8(7.3)(1.6)$ & $19.0(6.3)(6.0)$ & $23.2(3.2)(0.5)$ \\
48 & $27(14)(05)$ & $36(12)(04)$ & $16.6(6.9)(3.2)$ & $19.5(5.6)(2.3)$ \\
96 & $24(18)(12)$ & $24(14)(03)$ & $19.0(7.6)(4.9)$ & $18.4(6.1)(1.9)$ \\
$\infty$ & \multicolumn{2}{c}{$27.7(7.8)(5.5)$} & \multicolumn{2}{c}{$18.2(3.5)(2.9)$} \\
\hline \hline
\end{tabular}

Table 2: Energy shifts for ${ }^{4} \mathrm{He}$ and ${ }^{3} \mathrm{He}$ channels on each spatial volume. Extrapolated results to the infinite spatial volume limit are also presented. The first and second errors are statistical and systematic, respectively.

sources with a fit function of $\Delta E+C / L^{3}$ yields a finite value of $\Delta E=18.2(4.5) \mathrm{MeV}$, with the combined errors as in the ${ }^{4} \mathrm{He}$ channel, in the infinite volume limit. This means the existence of a bound state in the ${ }^{3} \mathrm{He}$ channel. Our result for $\Delta E$, however, is about twice larger than the experimental value of $7.72 \mathrm{MeV}$. The main reason could be the heavy pion mass employed in this calculation.

As an alternative way to view this result, we compare the binding energies normalized by the atomic number: $\Delta E / N_{N}=6.9(2.4) \mathrm{MeV}$ and 6.1(1.5) $\mathrm{MeV}$ for the ${ }^{4} \mathrm{He}$ and ${ }^{3} \mathrm{He}$ nuclei, respectively. At our unphysically heavy pion mass, the three and four nucleon systems do not show the experimental feature that the binding is stronger for ${ }^{4} \mathrm{He}$ than for ${ }^{3} \mathrm{He}$. We consider that this is mainly caused by the heavy quark mass used in this calculation.

\section{Toward further progress}

We have addressed the issue of nuclear binding for the case of ${ }^{4} \mathrm{He}$ and ${ }^{3} \mathrm{He}$ nuclei. We have shown that the current computational techniques and resources allow us to tackle this issue. Albeit in quenched QCD and for unphysically heavy pion mass, we are able to extract evidence for the bound state nature of the ground state and the binding energies for these nuclei.

It is encouraging that our results for the binding energies and the experimental values are of same order of magnitude. There are several issues which need clarification, however. Our conclusion for ${ }^{3} \mathrm{He}$ seems to disagree with the recent result of NPLQCD Collaboration [6, 19]. While the two simulations have been carried out under different parameters, e.g., quark mass, number of flavors, and volumes, further work is needed to obtain a consensus in this channel. Furthermore we should gain a deeper understanding on how the helium nuclei forms a bound state at such a heavy quark mass. Study of the nuclear force extracted from the wave function and looking at the simplest nucleus, deuteron, might help to understand our results.

A future direction of primary importance is to investigate the quark mass dependence of the binding energies of the nuclei. There are several model studies of the quark mass dependence of the nuclear binding energies [38] which suggest that the quark masses play an essential role in a quantitative understanding of the binding energies.

Another important issue is development of a strategy to calculate nuclei with larger atomic numbers. The number of Wick contractions quickly diverges as the atomic number increases, even if the redundancies are removed with various symmetries and techniques employed in this 
work. At this conference, NPLQCD Collaboration presented a set of recursion relations [25, 26] for correlation functions for the $n$-meson system, and also for more complex systems with multispecies, such as $n-\pi$ and $m-K$ systems. Similar recursion relations in multi-baryon systems might solve the problem. We leave it to future work.

\section{Acknowledgments}

We would like to thank the organizers of Lattice 2010 for the invitation of this presentation. Numerical calculations for the present work have been carried out on the HA8000 cluster system at Information Technology Center of the University of Tokyo and on the PACS-CS computer under the "Interdisciplinary Computational Science Program" of Center for Computational Sciences, University of Tsukuba. We thank our colleagues in the PACS-CS Collaboration for helpful discussions and providing us the code used in this work. This work is supported in part by Grants-in-Aid for Scientific Research from the Ministry of Education, Culture, Sports, Science and Technology (Nos. 18104005, 18540250, 20105002, 21105501, 22244018).

\section{References}

[1] M. G. Mayer, On Closed Shells in Nucei. II, Phys. Rev. 75 (1949) 1969.

[2] O. Haxel, J. H. D. Jensen, and H. E. Suess, On the "Magic Numbers" in Nuclear Structure, Phys. Rev. 75 (1949) 1766.

[3] R. L. Jaffe, Perhaps a Stable Dihyperon, Phys. Rev. Lett. 38 (1977) 195-198.

[4] P. B. Mackenzie and H. B. Thacker, Evidence Against a Stable Dibaryon from Lattice QCD, Phys. Rev. Lett. 55 (1985) 2539.

[5] M. Fukugita, Y. Kuramashi, H. Mino, M. Okawa, and A. Ukawa, An Exploratory study of nucleon-nucleon scattering lengths in lattice QCD, Phys. Rev. Lett. 73 (1994) 2176-2179, [hep-lat/9407012].

[6] S. R. Beane et. al., High Statistics Analysis using Anisotropic Clover Lattices: (II) Three-Baryon Systems, Phys. Rev. D80 (2009) 074501, arXiv:0905.0466].

[7] P. de Forcrand and M. Fromm, Nuclear Physics from lattice QCD at strong coupling, Phys. Rev. Lett. 104 (2010) 112005, arXiv:0907.1915.

[8] PACS-CS Collaboration, T. Yamazaki, Y. Kuramashi, and A. Ukawa, Helium Nuclei in Quenched Lattice QCD, Phys. Rev. D81 (2010) 111504(R), arXiv:0912.1383.

[9] Y. Iwasaki, T. Yoshie, and Y. Tsuboi, THE H DIBARYON IN LATTICE QCD, Phys. Rev. Lett. 60 (1988) 1371-1374.

[10] A. Pochinsky, J. W. Negele, and B. Scarlet, Lattice study of the H dibaryon, Nucl. Phys. Proc. Suppl. 73 (1999) 255-257, hep-lat/9809077].

[11] I. Wetzorke, F. Karsch, and E. Laermann, Further evidence for an unstable H-dibaryon?, Nucl. Phys. Proc. Suppl. 83 (2000) 218-220, [hep-lat/9909037].

[12] I. Wetzorke and F. Karsch, The H dibaryon on the lattice, Nucl. Phys. Proc. Suppl. 119 (2003) 278-280, [hep-lat/0208029]. 
[13] NPLQCD Collaboration, S. R. Beane et. al., High Statistics Analysis using Anisotropic Clover Lattices: (III) Baryon-Baryon Interactions, Phys. Rev. D81 (2010) 054505, arXiv: 0912 . 4243.

[14] M. Fukugita, Y. Kuramashi, M. Okawa, H. Mino, and A. Ukawa, Hadron scattering lengths in lattice QCD, Phys. Rev. D52 (1995) 3003-3023, [hep-lat/9501024].

[15] S. R. Beane, P. F. Bedaque, K. Orginos, and M. J. Savage, Nucleon nucleon scattering from fully-dynamical lattice QCD, Phys. Rev. Lett. 97 (2006) 012001, hep-lat/0602010].

[16] N. Ishii, S. Aoki, and T. Hatsuda, The nuclear force from lattice QCD, Phys. Rev. Lett. 99 (2007) 022001, [nucl-th/0611096].

[17] S. Aoki, T. Hatsuda, and N. Ishii, Theoretical Foundation of the Nuclear Force in QCD and its applications to Central and Tensor Forces in Quenched Lattice QCD Simulations, Prog. Theor. Phys. 123 (2010) 89-128, arXiv:0909.5585].

[18] S. Aoki, T. Hatsuda, and N. Ishii, Nuclear Force from Monte Carlo Simulations of Lattice Quantum Chromodynamics, Comput. Sci. Dis. 1 (2008) 015009, arXiv: 0805.2462].

[19] W. Detmold, Multi-hadron systems, PoS LAT2009 (2009) 008.

[20] K. Murano, non-locality of the nucleon-nucleon potential from Lattice QCD, PoS Lattice2010 (2010) 150.

[21] T. Inoue, Flavor structure of the baryon-baryon interaction from lattice QCD, PoS Lattice2010 (2010) 144, [arXiv:1011.1695].

[22] N. Ishii, An extention to the Luescher's finite volume method above inelastic thresholds (formalism), PoS Lattice2010 (2010) 145.

[23] K. Sasaki, Lattice QCD study of baryon-baryon interactions in the $(S, I)=(-2,0)$ system using the coupled-channel formalism, PoS Lattice2010 (2010) 157.

[24] T. Doi, The study of the Three Nucleon Force in full QCD Lattice calculations, PoS Lattice2010 (2010) 136, [arXiv:1011.0657].

[25] W. Detmold and M. J. Savage, A method to study complex systems of mesons in Lattice QCD, Phys. Rev. D82 (2010) 014511, arXiv: 1001.2768.

[26] W. Detmold, New developments in multi-meson systems, PoS Lattice2010 (2010) 100.

[27] G. P. Lepage, THE ANALYSIS OF ALGORITHMS FOR LATTICE FIELD THEORY, Invited lectures given at TASI'89 Summer School, Boulder, CO, Jun 4-30, 1989.

[28] M. Lüscher, Volume dependence of the energy spectrum in massive quantum field theories. 2. scattering states, Commun. Math. Phys. 105 (1986) 153-188.

[29] S. R. Beane, P. F. Bedaque, A. Parreno, and M. J. Savage, Two Nucleons on a Lattice, Phys. Lett. B585 (2004) 106-114, [hep-lat/0312004].

[30] S. Sasaki and T. Yamazaki, Signatures of S-wave bound-state formation in finite volume, Phys. Rev. D74 (2006) 114507, [hep-lat/0610081].

[31] S. R. Beane, W. Detmold, and M. J. Savage, n-Boson Energies at Finite Volume and Three-Boson Interactions, Phys. Rev. D76 (2007) 074507, [arXiv:0707.1670].

[32] Y. Iwasaki, Report No. UTHEP-118 (1983) (unpublished).

[33] CP-PACS Collaboration, A. Ali Khan et. al., Light Hadron Spectroscopy with Two Flavors of Dynamical Quarks on the Lattice, Phys. Rev. $\mathbf{D 6 5}$ (2002) 054505, hep-lat/0105015. 
[34] I. P. Omelyan, I. M. Mryglod, and R. Folk, , Comput. Phys. Commun. 151 (2003) 272-277.

[35] T. Takaishi and P. de Forcrand, Testing and tuning new symplectic integrators for hybrid Monte Carlo algorithm in lattice QCD, Phys. Rev. E73 (2006) 036706, [hep-lat/0505020].

[36] J. E. Beam, Orthogonal Classification of Alpha-Particle Wave Functions, Phys. Rev. 158 (1967) 907-916.

[37] M. Bolsterli and E. Jezak, Vector Harmonics for Three Identical Fermions, Phys. Rev. 135 (1964) B510-B515.

[38] V. V. Flambaum and R. B. Wiringa, Dependence of nuclear binding on hadronic mass variation, Phys. Rev. C76 (2007) 054002, [arXiv:0709.0077]. 